\title{
Luminescence from Liquid Helium Excited by Corona Discharges
}

\author{
Z.-L. Li, N. Bonifaci, F. Aitken, A. Denat \\ G2E.lab (CNRS and Joseph Fourier University). \\ 25 rue des Martyrs \\ 38042 Grenoble, France
}

\author{
K. von Haeften \\ University of Leicester \\ Department of Physics and Astronomy \\ University Road, Leicester, UK \\ and V. M. Atrazhev \\ Joint Institute for High Temperatures RAS \\ Izhorskaya St. 13, 2 \\ Moscow, Russia \\ and V. A. Shakhatov \\ Topchiev of Petrochemical Synthesis Institute RAS \\ Leninskii Prospect 29 \\ Moscow, Russia.
}

\begin{abstract}
Liquid helium (LHe) at $4.2 \mathrm{~K}$ was electronically excited using a corona discharge for both negative and positive high voltages. The experiments were carried out for different pressures in the range from 0.1 to $10 \mathrm{MPa}$ at constant temperature. The light emitted from the zone close to the tip was spectroscopically analyzed showing features from atoms and excimer helium. The shifts and widths of the observed lines and bands were found to depend on the applied hydrostatic pressure and on the tip polarity. Our analysis showed that classic pressure broadening theory cannot account for the observed widths and shifts rather than the presence of bubbles which surround single excited atoms and molecules. For positive tip polarities red shifted features distinct from pure $\mathrm{He}$ and $\mathrm{He}_{2}{ }^{*}$ were observed and tentatively assigned to "red satellites".
\end{abstract}

Index Terms -Helium spectroscopy, corona discharge, excimer helium.

\section{INTRODUCTION}

LIQUID helium is a fascinating substance with many peculiarities due to its highly quantum nature. A particular feature of liquid helium is its intense luminescence in the visible and near infrared spectral range. This luminescence has been observed from superfluid ${ }^{4} \mathrm{He}$ bombarded with energetic electrons [1-3], from liquid helium excited by a corona discharge [4-8] as well as from ${ }^{4} \mathrm{He}$ droplets excited by monochromatic synchrotron radiation [9-11]. After electronic excitation, Rydberg-type He atoms or excimer molecules are formed in liquid helium and a repulsive force between the Rydberg electron orbital and the surrounding ground state helium atoms is established. As a consequence the surrounding helium atoms are pushed away within a short time [12-13] creating a void around

Manuscript received on 31 October 2008, in final form 1 April 2009. the excited atoms $\mathrm{He}^{*}$ and molecules $\mathrm{He}_{2}{ }^{*}$. This void is often referred to as a bubble and it has typical radii between 10-14 $\AA$ depending on the electron's orbital radius [14]. Bubbles of similar type are well known to enclose electrons in liquid and even dense gaseous helium [15-17]. Within the confinement of these bubbles the perturbation by surrounding ground state helium atoms is low and the electronic life time of the excited atoms or excimers is almost similarly long as for free species in the vacuum. The remaining perturbation by the ground state helium atoms surrounding the bubble is nevertheless strong enough to cause broadening and wavelength shifts of the atomic and molecular lines and the magnitude of the width and shift was found to depend on the applied pressure. The hydrostatic pressure was also found to affect the line intensity distribution of the rotational spectrum of the confined $\mathrm{He}_{2}{ }^{*}$ as well as the total luminescence yield. 
Corona discharge represents a relatively simple and versatile way to produce and investigate electronic excitations and luminescence in liquid helium as it allows changes of pressure and density over a very wide range. However, the mechanisms involved in such a discharge in the liquid phase are complicated and involve electronic avalanches, energy transfer, formation of shock waves and bubbles, etc. Another complication is that the particle density in corona discharge is not uniform [18]: the region close to the tip has a high excitation density and thus resembles in many respects a plasma. It is a priori not clear whether under these conditions in the zone close to the tip the liquid state of helium is retained. To obtain a better understanding of these effects we have initiated a systematic spectroscopic investigation of liquid helium which is excited by corona discharges.

In this paper we report on the effect of the tip polarity on the spectra obtained in the liquid phase at $4.2 \mathrm{~K}$ as well as the effect of external hydrostatic pressure. We show that most spectral features are due to light emission from excited He atoms and excimers. The features are broadened and shifted depending on te hydrostatic pressure. Classical line broadening theory cannot explain the magnitudes of the line shifts and widths and we find that the most likely origin of the perturbation is the presence of bubbles around the emitting species similar to the observation by Dennis et al. [1] who used electron bombardment for the excitation of superfluid helium. We further show that additional red-shifted spectral features exist that cannot be explained by rotational line intensity distributions of thermalized excimer molecules. These features depend on the polarity of the corona discharge giving rise to a provisional assignment to 'red satellites' side-bands. The microscopic origin of these red satellites is presently unknown.

\section{EXPERIMENTAL TECHNIQUES}

Our experimental set up has been described elsewhere [4] and we only briefly mention its most important features. The liquid sample was produced from helium at the grade N 60 (Air Liquide) with an impurity concentration of about $0.1 \mathrm{ppm}$ of oxygen. The gas was further purified by a series of traps that were filled with a mixture of molecular sieves (3-10 $\AA$ ) and charcoal, activated under vacuum typically at $350^{\circ} \mathrm{C}$ for 3 days and finally immersed into liquid $\mathrm{N}_{2}$. The liquid helium sample was obtained by condensing purified helium gas into a copper beryllium coaxial cell. The cell included a point electrode and had a characteristic impedance of $50 \Omega$ when mounted into a cryostat and it also could withstand pressures up to $10 \mathrm{MPa}$. Before filling the cell was pumped to about $10^{-4}$ $\mathrm{Pa}$ using a turbo-molecular pump. Tungsten tips with a radius of $0.45 \mu \mathrm{m}$ and $2.5 \mu \mathrm{m}$ were prepared by electrolytic etching. The electrode spacing was $8 \mathrm{~mm}$. All metallic electrodes were supported by Macor insulators. In the cryostat the temperature of the liquid could be adjusted to $4.2 \mathrm{~K}$ at a constant pressure P. The high voltage from a stabilized dc power supply (Spellman RHSR/20PN60) was connected to the point electrode. Light emitted from the region close to the point electrode was analyzed by a spectrograph through a sapphire window. The spectrograph (Acton Research Corporation of
$300 \mathrm{~mm}$ focal length) was equipped with 3 plane gratings: one with $150 \mathrm{gr} . / \mathrm{mm}$ and two with $1200 \mathrm{gr} . / \mathrm{mm}$ that were blazed at $750 \mathrm{~nm}$ and $300 \mathrm{~nm}$, respectively. The 2D-CCDTKBUV/AR detector was located directly in the exit plane of the spectrograph. In order to reduce the dark current, the detector was cooled to a temperature of $153 \mathrm{~K}$ (dark current $<1$ electron/pixel/hour at $153 \mathrm{~K}$. The instrumental broadening measured by recording the profiles of argon lines from a low pressure discharge lamp was $\Delta \lambda_{\text {ins }}=0.12 \mathrm{~nm}$ for the 1200 gr./mm gratings.

\section{EXPERIMENTAL RESULTS}

\subsection{GENERAL REMARKS}

The light emitted from the corona region was collected and spectra in the range $500-1080 \mathrm{~nm}$ were recorded. Figure 1 shows a representative spectrum observed in LHe. Several atomic lines and molecular bands can be identified. These lines correspond to radiative transitions between excited states of $\mathrm{He}^{*}$ atoms and $\mathrm{He}_{2}{ }^{*}$ excimer molecules.

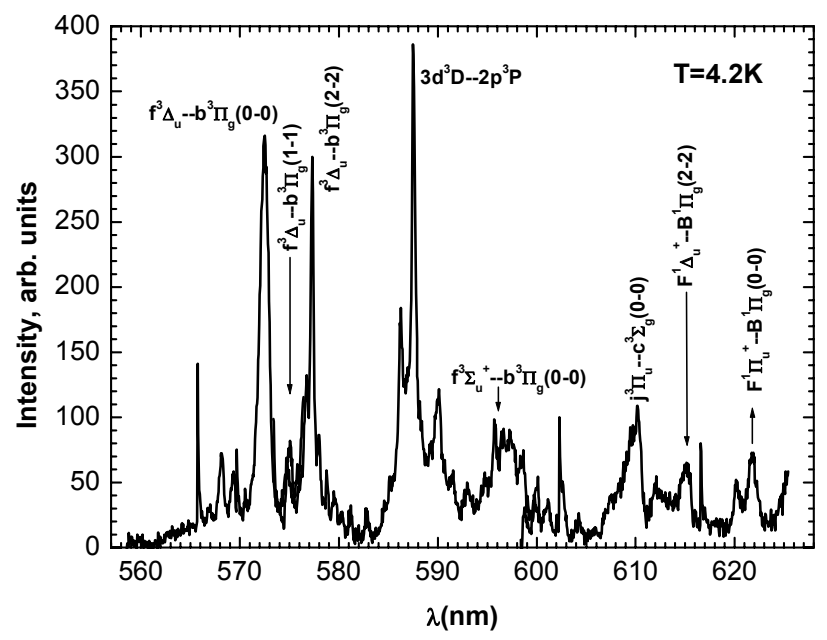

Figure 1. Overview spectrum recorded in the range 560-630 $\mathrm{nm}$ in LHe at 4.2 $\mathrm{K}, 0.1 \mathrm{MPa}$.

At low pressure the lines are sharp and their peak position match the atomic lines and molecular bands of helium from gas phase experiments. These lines are listed in Table 1.

A strong background continuum from 490 to $1100 \mathrm{~nm}$ appears in spectra at high pressures above $\mathrm{P}=4.0 \mathrm{MPa}$. Moreover, the width of the lines increases with pressure and their relative intensity decreases. Figure 2 shows the atomic $3 \mathrm{~s}^{3} \mathrm{~S}->2 \mathrm{p}^{3} \mathrm{P}$ line at $706 \mathrm{~nm}$ being broadened and shifted with increasing pressure towards smaller wavelengths (blue shift). The relative intensity of the line decreases with pressure. No lines and bands can be observed in spectra if the pressure exceeds 5.0 MPa.

Owing to the relatively small mass of He atoms the excimer spectrum of He exhibits a spectrum with comparatively large rotational line separation which our spectrometer is able to resolve. Figure 3 shows that for low pressures rotational lines 
can indeed be observed allowing us to derive the population of upper rotational levels of the excited molecules. For the case that the intensities are governed by a Boltzmann distribution it is possible to assign a "rotational temperature".

Table 1. Transitions observed in liquid helium $(\mathrm{T}=4.2 \mathrm{~K}, \mathrm{P}=0.1 \mathrm{MPa})$

\begin{tabular}{cccc}
\hline \multicolumn{2}{c}{ Atomic lines } & \multicolumn{2}{c}{ Molecular bands } \\
\hline$\lambda(\mathrm{nm})$ & Upper-Lower & $\lambda(\mathrm{nm})$ & Upper-Lower \\
\hline 492,19 & $4 \mathrm{~d}^{1} \mathrm{D}-2 \mathrm{p}{ }^{1} \mathrm{P}$ & 462,24 & $\mathrm{~J}^{1} \Delta_{\mathrm{u}}-\mathrm{B}^{1} \Pi_{\mathrm{g}}$ \\
587,56 & $3 \mathrm{~d}^{3} \mathrm{D}-2 \mathrm{p}{ }^{3} \mathrm{P}$ & 464,95 & $\mathrm{e}^{3} \Pi_{\mathrm{g}}-\mathrm{a}^{3} \Sigma_{\mathrm{u}}^{+}$ \\
706,52 & $3 \mathrm{~s}^{3} \mathrm{~S}-2 \mathrm{p}{ }^{3} \mathrm{P}$ & 573,49 & $\mathrm{f}^{3} \Delta_{\mathrm{u}}(\mathrm{v}=0)-\mathrm{b}^{3} \Pi_{\mathrm{g}}(\mathrm{v}=0)$ \\
728,13 & $3 \mathrm{~s}^{1} \mathrm{~S}-2 \mathrm{p}{ }^{1} \mathrm{P}$ & 575 & $\mathrm{f}^{3} \Delta_{\mathrm{u}}(\mathrm{v}=1)-\mathrm{b}^{3} \Pi_{\mathrm{g}}(\mathrm{v}=1)$ \\
1083,02 & $2 \mathrm{p}^{3} \mathrm{P}-2 \mathrm{~s}^{3} \mathrm{~S}$ & 577 & $\mathrm{f}^{3} \Delta_{\mathrm{u}}(\mathrm{v}=2)-\mathrm{b}^{3} \Pi_{\mathrm{g}}(\mathrm{v}=2)$ \\
& & 588,7 & $\mathrm{f}^{3} \Pi_{\mathrm{u}}{ }^{-} \mathrm{b}^{3} \Pi_{\mathrm{g}}$ \\
& & 639,6 & $\mathrm{~d}^{3} \Sigma_{\mathrm{u}}{ }^{+}-\mathrm{b}^{3} \Pi_{\mathrm{g}}$ \\
& & 659,55 & $\mathrm{D}^{1} \Sigma_{\mathrm{u}}{ }^{+}-\mathrm{B}^{1} \Pi_{\mathrm{g}}$ \\
& & 913,61 & $\mathrm{C}^{1} \Sigma_{\mathrm{g}}{ }^{+}-\mathrm{A}^{1} \Sigma_{\mathrm{u}}{ }^{+}$ \\
& & 918,3 & $\mathrm{c}^{3} \Sigma_{\mathrm{g}}{ }^{+}-\mathrm{a}^{3} \Sigma_{\mathrm{u}}{ }^{+}$ \\
\hline
\end{tabular}

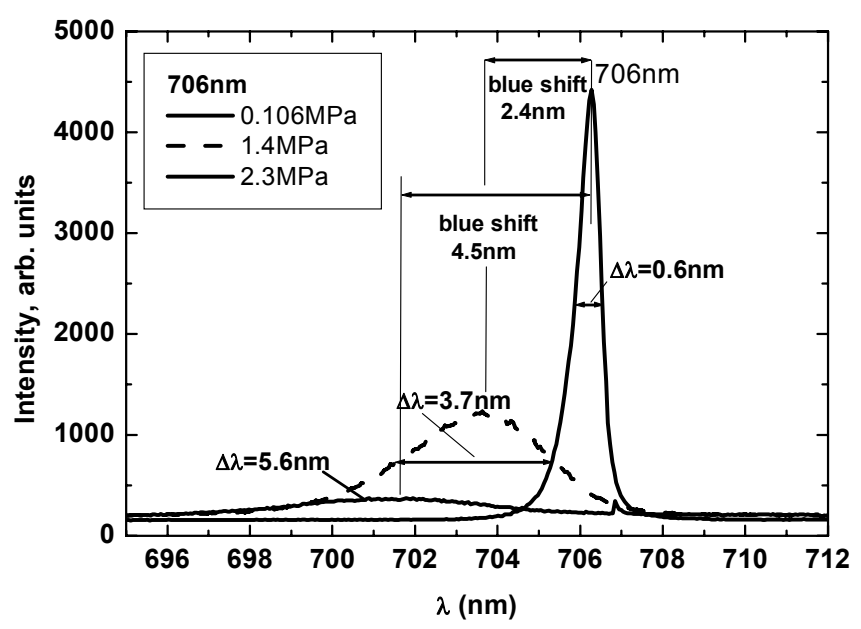

Figure 2. Intensity of the $706 \mathrm{~nm}$ atomic line for different pressures. LHe at $4.2 \mathrm{~K}, \mathrm{I}_{\text {corona }}=0.3 \mu \mathrm{A}$, Power $=1 \mathrm{~mW}$.

In gaseous discharges of molecular species the rotational temperature is often close to the thermodynamic temperature of the gas, however, exceptions especially for helium have been reported [19]. The different behavior of $\mathrm{He}$ is due to the excimer molecules being formed after the electronic excitation. Likewise, the rotational temperatures in the molecular bands observed in $\mathrm{LHe}$ or He droplets were by two orders of magnitude higher than the temperature of the liquid or the droplets [1].

In the following we will investigate the effect of hydrostatic pressure on the spectra and we will also investigate the effect of the polarity of the discharge. Increased hydrostatic pressure leads to broadening and shifts of the lines. The spectra recorded with positive tip polarity show distinct differences in the form of red-shifted features. These features were observed near atomic and molecular lines.

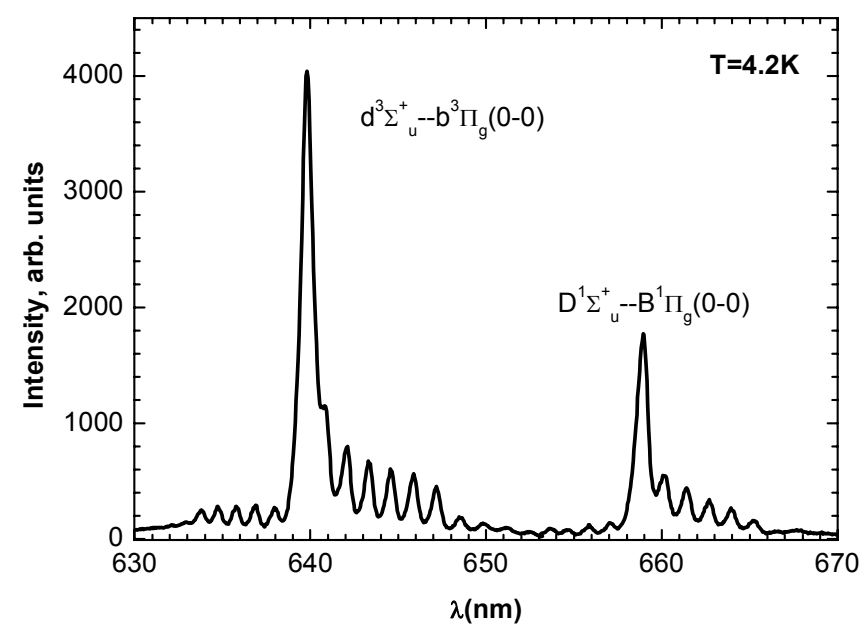

Figure 3. Molecular features in the spectrum of LHe at $4.2 \mathrm{~K}$ and $0.1 \mathrm{MPa}$ showing rotational structure.

\subsection{SPECTRA FROM NEGATIVE CORONA DISCHARGES}

In this section, we show atomic and molecular spectra recorded with negative tip polarities. Figure 4 shows the same spectrum of the $3 s^{1} S->2 p^{1} P$ atomic transition at $706 \mathrm{~nm}$ as in Figure 2 but with normalized line intensity. In this way it can be seen that changes in pressure up to $1.4 \mathrm{MPa}$ cause increased line widths, but with no significant changes in the symmetry of the line. Further increments in pressure up to $2.3 \mathrm{MPa}$ produce slightly asymmetric line shapes. The retained symmetric character of the line allowed us to quantify the width using the magnitude of the full width at half maximum (fwhm). The magnitude of the shift was derived from the position of the maximum.

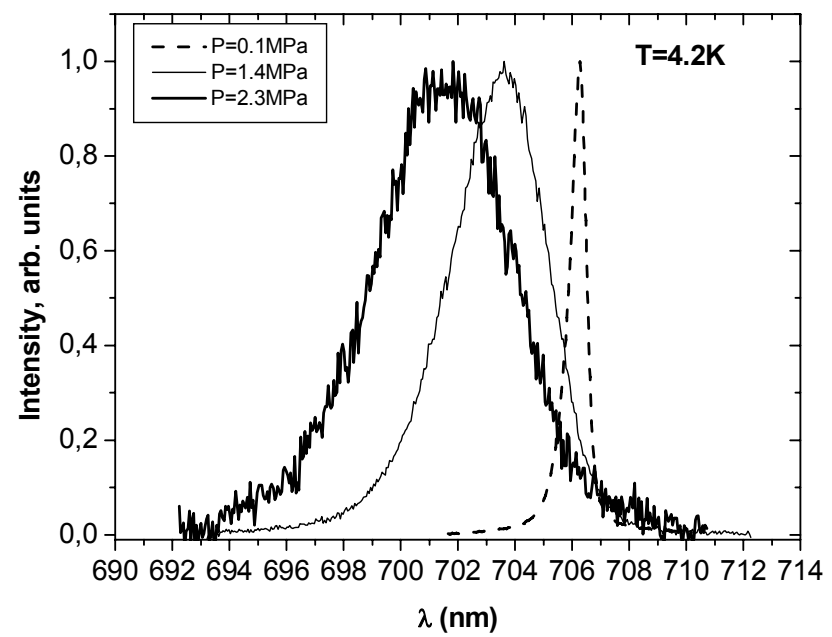

Figure 4. Normalized intensities of the $706 \mathrm{~nm}$ atomic line for different pressures. LHe at $4.2 \mathrm{~K}$.

In Figure 5 we show the dependence of the shift and width on the pressure. Both show a linear dependence on the 
pressure within the accuracy of our measurements. For high pressures the widths and the shifts have similar magnitudes which are remarkably distinct from the behavior of dense $\mathrm{He}$ gas at room temperature.

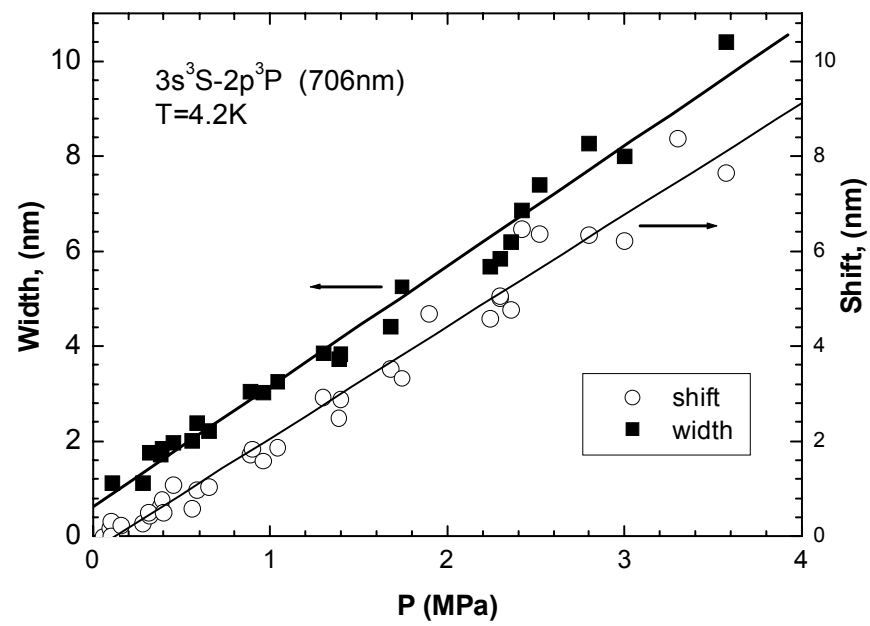

Figure 5. Shift and broadening of the $706 \mathrm{~nm}$ atomic line vs. pressure. LHe at $4.2 \mathrm{~K}$. Lines are linear fitting of the data curves comparing.

The molecular bands observed in our experiments with the negative corona show broadening and shifts with increasing pressure as well. The spectrum of the $d^{3} \Sigma_{u}^{+}-b^{3} \Pi_{g}$ triplet transition at $640 \mathrm{~nm}$ is shown in Figure 6 for three different pressures.

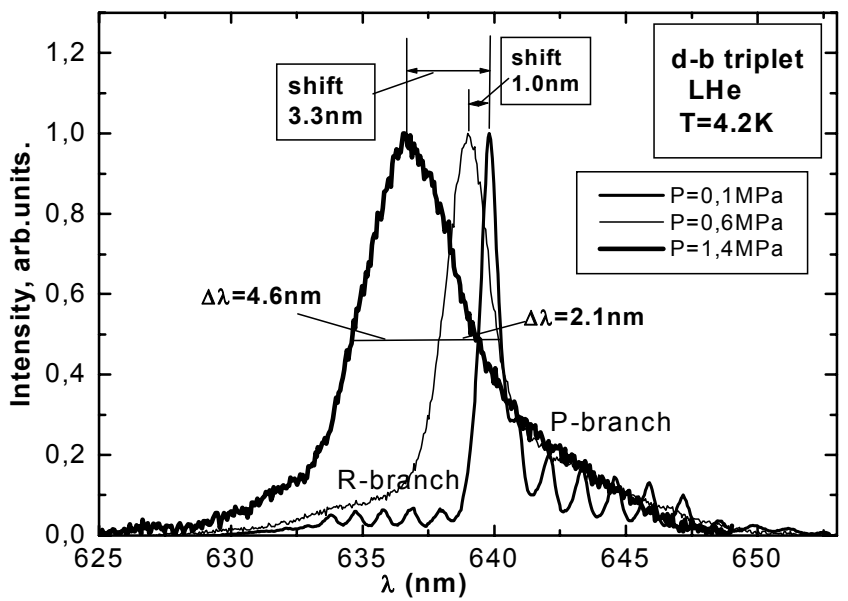

Figure 6. Transition $d^{3} \Sigma^{+}{ }_{u}-b^{3} \Pi_{g}$ in LHe at $4.2 \mathrm{~K}$ for different pressures. The intensities are normalized.

As for the atomic lines the spectral features shift to shorter wavelengths with increasing pressure and become more broadened. The rotational structure of the band is resolved at $0.1 \mathrm{MPa}$ and shows the $\mathrm{R}, \mathrm{Q}$, and $\mathrm{P}$ transitions due to changes of the rotational quantum number $\Delta \mathrm{N}=1,0,-1$, respectively. We note that the spacing of the Q-lines is too close to be resolved with our spectrometer. The widths of the $\mathrm{R}$ and $\mathrm{P}$ rotational lines are $0.7 \mathrm{~nm}$ at $0.1 \mathrm{MPa}$. With increased pressure the widths of the lines quickly becomes larger than the $1.3 \mathrm{~nm}$ wide spacing of the rotational $\mathrm{P}$ and $\mathrm{R}$ lines which makes the analysis very difficult. We note that the profile of the band recorded at $0.6 \mathrm{MPa}$ is very similar to the spectrum reported by Dennis et al. who bombarded superfluid helium with high energetic electrons [1]. The shift of the Q-branch maximum with pressure is presented in Figure 7. Experimental data [2] obtained in superfluid $\mathrm{He}$ II at $1.7 \mathrm{~K}$ are also shown for comparison.

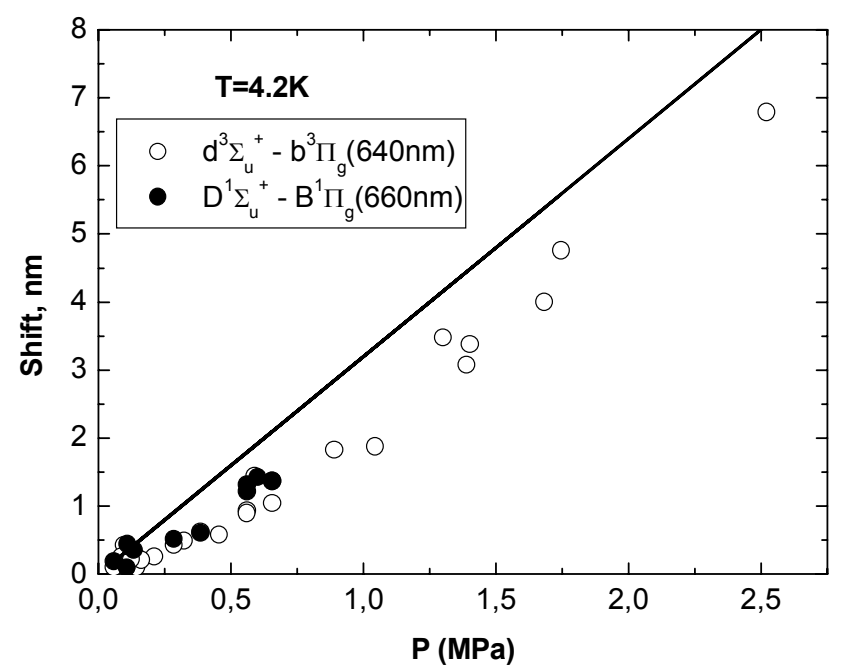

Figure 7. Shift of molecular bands for different pressures. The circles represent our experimental data whereas the line shows the shift reported by Soley and Fitzsimmons [2]. For pressures larger than 0.2 MPa the rotational lines overlap strongly and therefore the widths cannot be analysed.

\subsection{SPECTRA FROM POSITIVE CORONA}

Below, we show spectra recorded with positive tip polarity. These spectra show both atomic lines and molecular bands at $4.2 \mathrm{~K}$ over a wide range of pressures but very different line shapes compared to those obtained with negative tip polarities. Figures 8 and 9 show the atomic $1 \mathrm{~s}^{3} \mathrm{~s}^{3} \mathrm{~S}_{1}->$ $1 \mathrm{~s} 2 \mathrm{p}^{3} \mathrm{P}_{0,1,2}$ line at $706 \mathrm{~nm}$ and the $1 \mathrm{~s} 3 \mathrm{~s}^{1} \mathrm{~S}_{0}->1 \mathrm{~s} 2 \mathrm{p}^{1} \mathrm{P}_{1}$ transition at $728 \mathrm{~nm}$ at $4.2 \mathrm{~K}$ and $0.1 \mathrm{MPa}$. For negative tip polarity under same conditions these lines reveal no shift and no broadening (Figure 9), but in a positive corona discharge the lines clearly show a strongly asymmetric profile which can be fitted by a superposition of two Gaussian functions shifted from each other (Figure 10). The first, non-shifted feature has a width $\Delta \lambda_{1}=0.706 \mathrm{~nm}$. The second feature is shifted by 1.6 $\mathrm{nm}$ towards lower wavelengths and has a larger width $\Delta \lambda_{2}=$ $3.3 \mathrm{~nm}$.

Figure 11 shows the molecular $d^{3} \Sigma^{+}{ }_{u}-b^{3} \Pi_{g}$ transition of $\mathrm{He}_{2}$ using positive tip polarity. Similar to the atomic lines this band shows an asymmetric profile. In order to assess the degree of deviation we simulated the pure molecular $\mathrm{d}^{3} \Sigma^{+}{ }_{\mathrm{u}}$ $\mathrm{b}^{3} \Pi_{\mathrm{g}}$ transition (Figure 11) and we subtracted the simulated spectrum from the measured features. Figure 11 shows the resulting difference spectrum. This difference spectrum shows a clear asymmetry with regard to the pure molecular band and cannot be explained by broadening of by shifting of lines because $\mathrm{P}$ and $\mathrm{R}$ lines would be equally affected. The clear distinction from the well-known feature of pure $\mathrm{He}_{2}$ molecules gives rise to assign the difference spectrum to a "red-satellite" of unknown origin. 


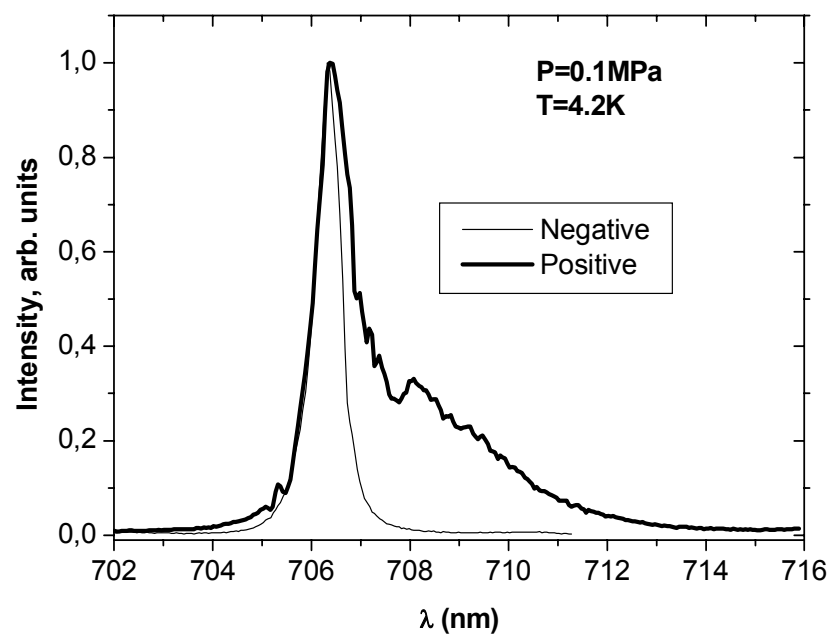

Figure 8. Atomic line of 706nm of negative and positive coronas in $\mathrm{LHe}, \mathrm{T}=$ $4.2 \mathrm{~K}, \mathrm{P}=0.1 \mathrm{MPa}$.

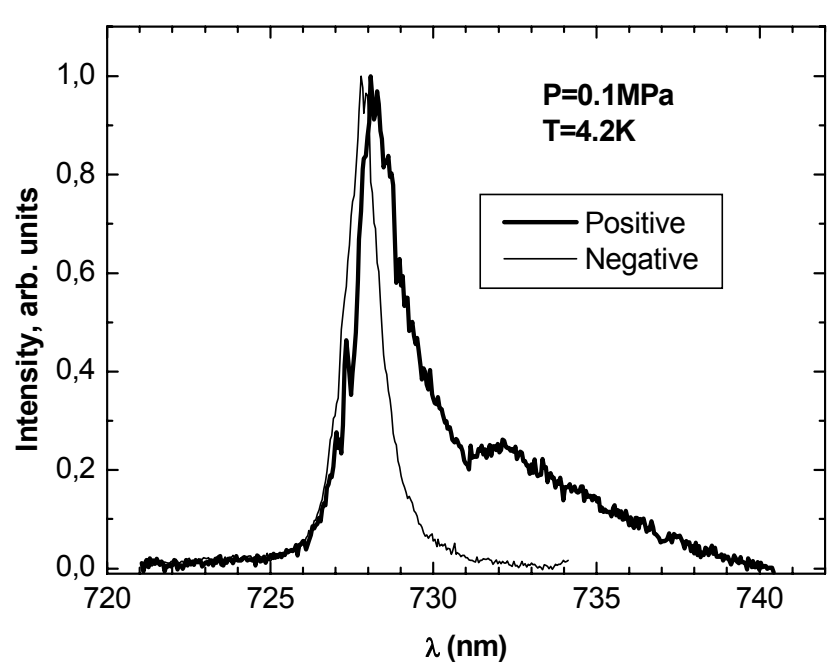

Figure 9. Atomic line at $728 \mathrm{~nm}$ for negative and positive corona discharge excitation in $\mathrm{LHe} ; \mathrm{T}=4.2 \mathrm{~K}, \mathrm{P}=0.1 \mathrm{MPa}$.

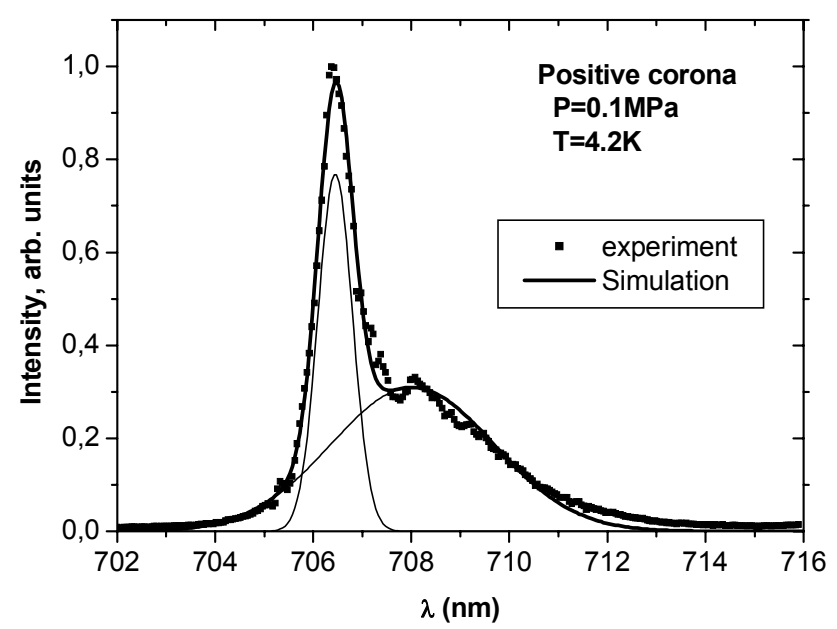

Figure 10. Atomic line of $706 \mathrm{~nm}$ of positive corona in LHe simulated as superposition of two Gaussian profiles.

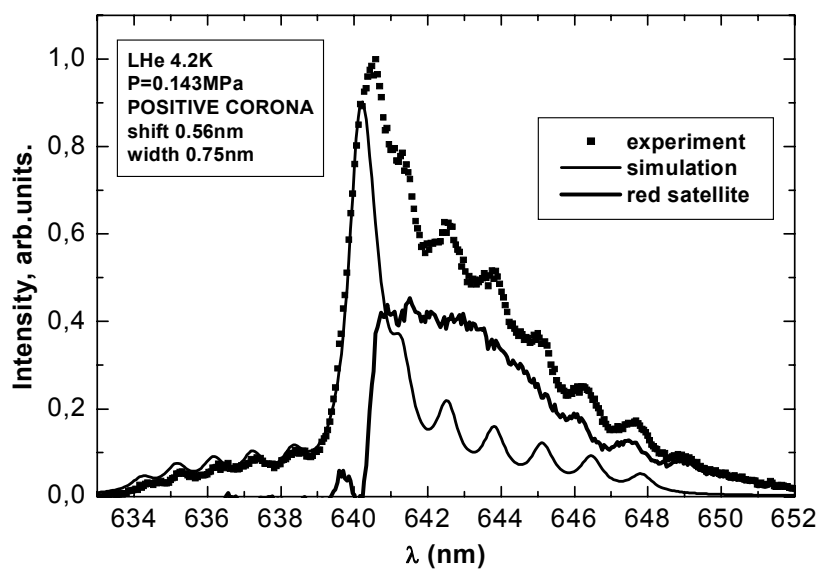

Figure 11. Rotational spectra of transition $\mathrm{d}^{3} \Sigma^{+}{ }_{\mathrm{u}}-\mathrm{b}^{3} \Pi_{\mathrm{g}}$ of $\mathrm{He}_{2}$ in $\mathrm{LHe}, \mathrm{T}=4.2$ $\mathrm{K}, \mathrm{P}=0.143 \mathrm{MPa}$.

\section{DISCUSSION}

The theory of the pressure broadening of spectral lines using so called "collision approach" is usually adopted to account for the line shapes observed in gaseous discharges [20]. Strictly speaking, the collision approach is only valid for low density gases but LHe may be an exception because it has the lowest density of all condensed matter and we therefore investigate its applicability.

The collision approach predicts symmetric Lorentzian line profiles with widths being proportional to the gas pressure. In helium gas the dependence of the line widths and shifts on the pressure are dominated by the repulsive interaction $(\mathrm{V}(\mathrm{r})=$ $\mathrm{C}_{12} \mathrm{r}^{-12}$ ) between an excited atom and surrounded atoms [21]. Within the so-called "limit of collision" for repulsive interaction, the expressions for the line broadening and the line shift are given by:

$$
\begin{aligned}
& \Delta \lambda_{12}=6.44\left(\frac{\lambda_{u l}^{2}}{2 \pi c}\right) w^{9 / 11}\left|C_{12}\right|^{2 / 11} N \\
& \Delta S_{12}=0.922\left(\frac{\lambda_{u l}{ }^{2}}{2 \pi c}\right) w^{9 / 11}\left|C_{12}\right|^{2 / 11} N
\end{aligned}
$$

Here $\lambda_{\mathrm{ul}}$ is the wavelength of the line in [m], $\mathrm{w}=10^{4} * \mathrm{~T}^{0.5}$ is the relative velocity of the gas atoms with $\mathrm{T}$ being the gas temperature in $[\mathrm{K}], \mathrm{C}_{12}$ is the repulsive Lennard-Jones parameter in $\left[\mathrm{m}^{12} \mathrm{~s}^{-1}\right]$ and $\mathrm{N}$ is the gas number density in $\left[\mathrm{m}^{-}\right.$ $\left.{ }^{3}\right]$. Regardless of the type of gas the collision approximation predicts a ratio between the shift and the width of 0.143 which is indeed close to what we find in our experiments with $\mathrm{He}$ gas at $300 \mathrm{~K}$.

A consequence of the dominating repulsive interaction in $\mathrm{He}$ is that the lines are shifted towards shorter wavelengths. With increasing gas density the atomic He lines become more asymmetric in shape. This deviation from the initially symmetric blue-shifted lines can be accounted by the so-called 
"static approximation" [22]. We point out that when using positive tip polarity we did not observe any "red satellites" in the atomic line spectrum in gaseous Helium at $300 \mathrm{~K}$.

In contrast to the gas phase we observe that in liquid helium the shift/width ratio is no longer constant and shows a distinct pressure dependence. For high pressures we find a value of about 0.8 for this ratio. For low pressures the shift/width ratio is lower but it never assumes a value of 0.143 . The proximity of the shift/width ratio to the theoretical prediction may be taken as a measure for the applicability of the classical pressure broadening theory using the collision approach but we emphasise that deviations simply may indicate that other physical processes become relevant.

Previous theoretical and experimental studies on electron beam bombarded liquid helium have provided convincing evidence for the existence of microscopic cavities or "bubbles" having a diameter of about $1 \mathrm{~nm}$ which enclose excited atoms and excimer molecules within liquid helium $[14,23]$. The origin of bubbles around an excited state of an atom or a molecule results from the balance between the repulsive interaction between the Rydberg electron and the ground state $\mathrm{He}$ atoms arising from the Pauli principle on the one hand and the pressure and the surface tension of liquid on the other hand. The shift of the spectral lines and their width depend primarily on the size of the bubble which in turn results from the balance between the repulsive force from the Rydberg electron and force on the bubble surface exerted by the hydrostatic pressure. Therefore the bubble size is pressure dependent.

It is a reasonable assumption that light emission from molecules enclosed in bubbles occurs in corona dischargeexcited LHe as well and the similarity between our molecular spectra recorded at $0.6 \mathrm{MPa}$ and $4.2 \mathrm{~K}$ with negative tip polarity and those reported by Dennis and coworkers [1] supports this interpretation. The pressure dependence seen in our spectra of LHe are therefore likely due to the perturbation exerted by the presence of bubbles. When the bubble size decreases with pressure the perturbation of the dominating upper level of the light emitting atom or molecule increases because the average distance to surrounding ground atoms becomes smaller. The line broadening theory which is valid for gaseous Helium cannot be applied anymore because it assumes the medium around the perturbed species to be homogeneous. This is no longer the case for a bubble. In Figure 12 we have also compared the measured widths of the $706 \mathrm{~nm}$ atomic line with the widths predicted by the "bubble" theory developed by Hickman et al. [14]. While there is agreement for small pressures deviations become significant at pressures larger than 1.0 MPa.

We note further that the bubble model of the spectral line shape predicts larger shifts than widths. The shift/width ratio calculated in [14] is close to 3 which is significantly larger than that predicted for "collision broadening" in gaseous discharges which can be taken as a major attribute of the spectrum of an excited atom inside a cavity in LHe.

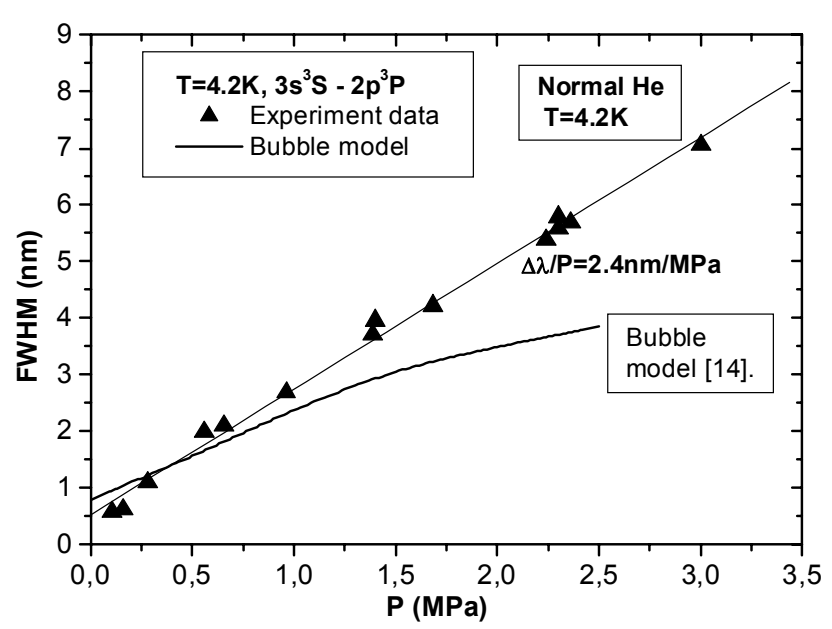

Figure 12. Full-Width-at-Half-Maximum (FWHM) of the $706 \mathrm{~nm}$ atomic line vs. pressure. $\mathrm{LHe}$ at $4.2 \mathrm{~K}$.

\subsection{RED SATELLITES IN NEGATIVE CORONA}

A very small contribution of "red satellites" to molecular spectral features can be found in negative corona discharges as well. We have simulated the population distribution of the upper rotational levels for $\mathrm{P}=0.1 \mathrm{MPa}$, where the rotational structure of the band is still clearly resolved (Figure 13). A Boltzmann distribution corresponds to a linear function in this semi-logarithmic plot. The plot shows that a Boltzmann distribution is only seen for large $\mathrm{N}$. The "rotational temperature" derived from this distribution is $780 \mathrm{~K}$ which is significantly higher than the temperature of $4.2 \mathrm{~K}$ of the liquid. The populations derived from the P-branch intensities are larger than those from R-branch intensities. This fact can formally be interpreted by the existence of an additional source of radiation that contributes a side-band at larger wavelengths to the spectrum. A simulation of the d-b spectrum in Figure 14 shows the magnitude of the contribution of such a "red satellite" band as the difference between measured and calculated spectra.

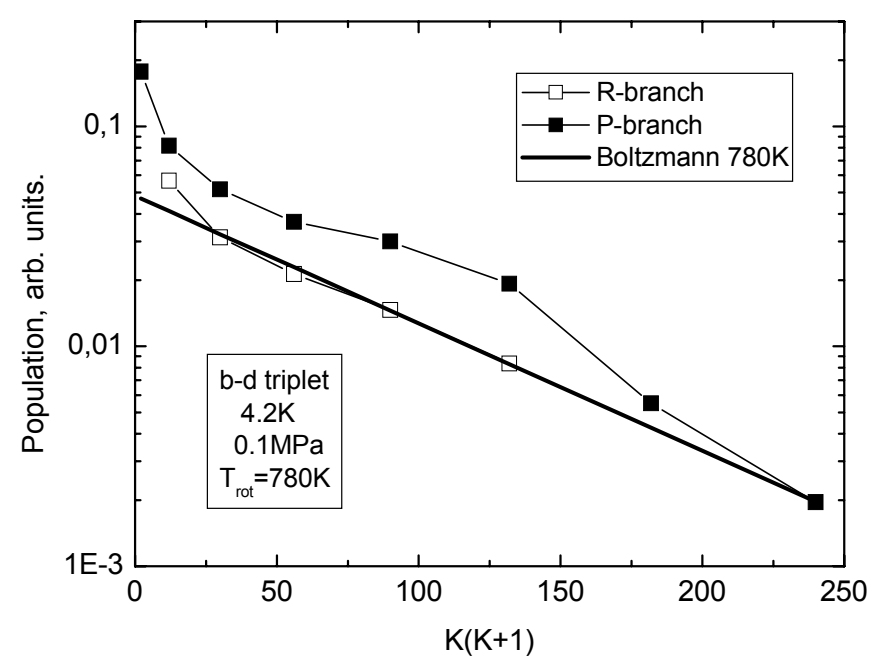

Figure 13. Relative population of the rotational levels of the upper term $\mathrm{d}^{3} \Sigma^{+}{ }_{\mathrm{u}}$ $-b^{3} \Pi_{g}$ (idem to Figure 6) for negative corona. 


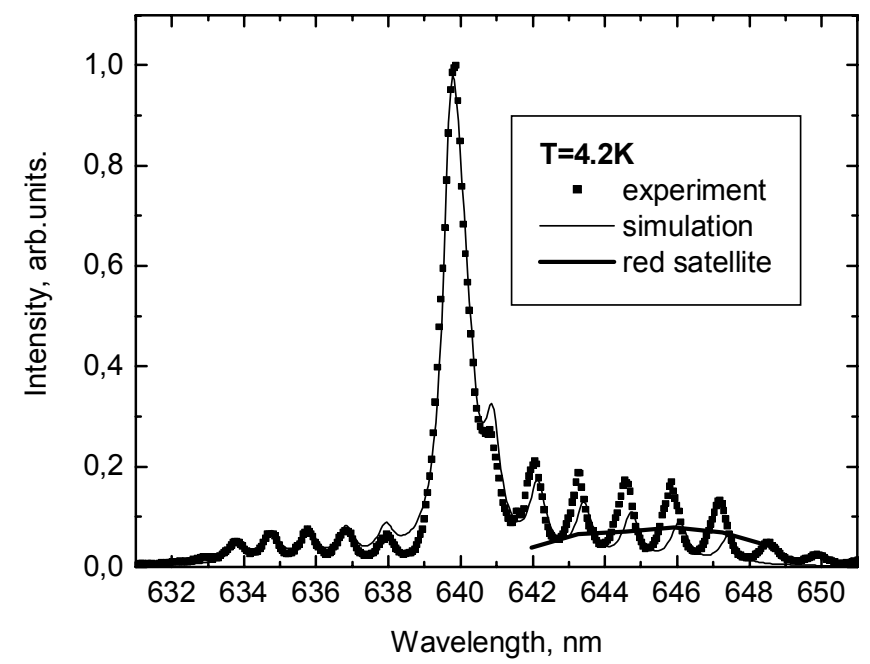

Figure 14. Rotational spectra of the $d^{3} \Sigma^{+}{ }_{u}-b^{3} \Pi_{g}$ transition: experiment, simulation and "red satellite" in LHe.

\subsection{RED SATELLITES IN POSITIVE CORONA}

As stated before the intensities of the "red satellite" bands are much stronger in spectra recorded with positive corona discharges. A similar plot of the population distribution of the upper rotational levels to the one in Figure 14 is shown in Figure 15.

It is difficult to give a definite explanation for the nature of the red satellites. We have explicitly checked whether the "red satellite" stems from higher vibrational levels such as the $\mathrm{v}^{\prime}=1$ $>\mathrm{v}^{\prime \prime}=1$ transition whose band head is located close to $642 \mathrm{~nm}$ and we found that this possibility has to be excluded. The strong tip polarity dependence suggests that charge carriers are involved. To make matters worse an explanation of the phenomenon of positive corona discharges has to the best of our knowledge not been reported yet, although its existence is well known. Clearly, a positive tip polarity raises the question as to how the electrons are released from the tip. It might be possible that free electrons are generated by entirely different processes, for instance, collisions with high energetic cosmic particles and that the role of the tip is only to generate high electric field strengths which eventually causes avalanches. The resulting excitation densities can therefore differ depending on the polarity. The asymmetric profile of the red satellite bands can be due to vibrational states of a van der Waals complex formed by the radiating atom or molecule and a helium gas atom in the ground state. We also recall the red-satellite features are only seen at $4.2 \mathrm{~K}$, but not for gaseous $\mathrm{He}$ at $300 \mathrm{~K}$.

Due to the many unknowns we cannot offer a conclusive explanation for the precise origin of the "red satellites".

\section{CONCLUSION}

We have shown that corona discharges are well-suited to excite visible luminescence of liquid helium at $4.2 \mathrm{~K}$ and to investigate its pressure dependence. The luminescence spectrum shows lines from transitions between electronically excited states of helium atoms and excimer molecules. For

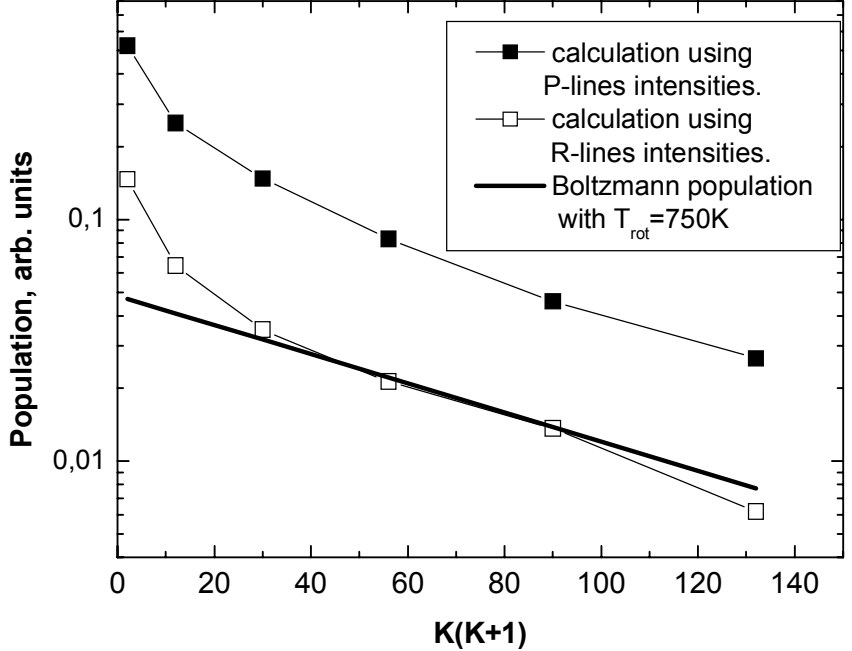

Figure 15. Relative population of the rotational levels of the upper term $\mathrm{d}^{3} \Sigma^{+}{ }_{\mathrm{u}}$ - $b^{3} \Pi_{g}$ (idem to Figure 11) for positive corona.

negative tip polarity and increasing hydrostatic pressure the lines become broader and shift towards shorter wavelengths.

For positive tip polarities a similar line spectrum can be observed, however, all lines are accompanied by red-shifted features. Also, the lines show much larger broadening than for negative tip polarities at similar pressure. Comparison between the $\mathrm{P}$ and $\mathrm{R}$ lines within molecular luminescence bands shows that the red-shifted features cannot originate from He excimer molecules and that they cannot be caused by a broadening process because $\mathrm{P}$ and $\mathrm{R}$ lines would then be equally affected. Therefore, the additional component in the spectrum is tentatively assigned to the contribution of an unknown "red-satellite". Since the underlying physical mechanisms of positive corona discharges are unknown it is not possible to further clarify the precise nature of the satellite spectrum on the basis of the available data.

Furthermore, we have investigated the dependence of the magnitudes of shift and broadening on the pressure for negative corona discharges. Classic line broadening theory predicts that these quantities are connected with each other such that the ratio between shift and width is a constant of 0.143 . Our analysis of the spectral features of liquid $\mathrm{He}$ at 4.2 $\mathrm{K}$ reveals a higher ratio indicating that classic pressure broadening theory cannot be applied. We presume that the emitting atoms and molecules are localized in bubbles similar to the observations by Dennis et al., Soley and Fitzsimmons $[1,2]$. The similarity between our spectral features and their reported spectral data supports our interpretation.

\section{ACKNOWLEDGMENT}

V. M. Atrazhev and V. A. Shakhatov are grateful to the Russian Foundation of Basic Researches who supported this work under the grant 08-08-00694. K. von Haeften acknowledges support by the British Council through a RXP award. 


\section{REFERENCES}

[1] W.S. Dennis, E. Durbin, Jr., W. A. Fitzsimmons, O. Heybey, and G. K. Walters, "Spectroscopic identification of excited atomic and molecular states in electron-bombarded liquid helium", Phys. Rev. Lett., Vol. 23, pp.1083-1086, 1969.

[2] F.J. Soley and W.A. Fitzsimmons, "Pressure shifts and quenching of atomic and molecular states produced in electron-bombarded liquid helium", Phys. Rev. Lett., Vol. 32, pp. 988-991, 1974.

[3] J.W. Keto, F.J. Soley, M. Stockton and W.A. Fitzsimmons, "Dynamic properties of neutral excitation produced in electron-bombarded superfluid helium. I. the $\mathrm{He}(23 \mathrm{~S})$ and $\mathrm{He} 2(\mathrm{a} 3 \mathrm{~S})$ atomic and molecular metastable states", Phys. Rev. A, Vol. 10, pp. 872-885, 1974.

[4] Z. Li, N. Bonifaci, A. Denat and V. Atrazhev, "Ionization phenomena in liquid helium by the corona discharge", IEEE Trans. Dielectrics Electrical Insulation, Vol. 13, pp. 624-631, 2006.

[5] P.H. Zimmermann and J.F. Reichert, "Study of the electron spin resonance of negative ions field emitted into liquid helium”, Phys. Rev. B, Vol. 15, pp. 2630-2650, 1977.

[6] V.A. Goncharov and V.I. Levitov, "Corona discharge and electrical breakdown in helium at non-uniform field at temperatures $4.2 \mathrm{~K}-10 \mathrm{~K}$ at pressure 1-10atm", Izvestiya Academii Nauk, Energetika i Transport (in Russian) Vol. 12, pp. 134-139, 1975.

[7] S.G. Kafanov, A.Ya. Parshin, I.A. Todoshenko, "Structure and Dynamics of the Molecular Complex $\mathrm{He}_{2}{ }^{*}\left(\mathrm{a}^{3} \Sigma^{+}{ }_{\mathrm{u}}\right)$ in the Condensed Phases of Helium", Sov. Phys. JETP, Vol. 91, pp. 1143-1152, 2000.

[8] A.Ya. Parshin, I.A. Todoshenko, S.G. Kafanov, "Optical spectra of the triplet $\mathrm{He}_{2}{ }^{*}$ excitations in various phases of liquid ${ }^{3} \mathrm{He}$ and ${ }^{4} \mathrm{He}$ under high pressures", Physica. B, Vol. 284-288, pp. 91-92, 2000.

[9] K. von Haeften, A. R. B. de Castro, M. Joppien, L. Moussavizdeh, R. von Pietrowski and T. Möller, "Discrete Visible Luminescence of Helium Atoms and Molecules Desorbing from Helium Clusters: The role of electronic vibrational and rotational energy transfer", Phys. Rev. Lett., Vol. 78, pp. 4371-4374, 1997.

[10] K. von Haeften, T. Laarmnn, H. Wabnitz and T. Möller, "Bubble formation and decay in ${ }^{3} \mathrm{He}$ and ${ }^{4} \mathrm{He}$ clusters", Phys. Rev. Lett., Vol. 88 , pp. 233401-4, 2002.

[11] K. von Haeften, T. Laarmnn, H. Wabnitz and T. Möller, "The electronically excited states of helium clusters: an unusual example for the presence of Rydberg states in condensed matter", J. Phys. B: Atomic Molecular Opt. Phys., Vol. 38, pp. S373-S386, 2005.

[12] J. Eloranta and V. Apkarian, "A time dependent density functional treatment of superfluid dynamics: Equilibrium of the electron bubble in superfluid ${ }^{4} \mathrm{He}$ ", J. Chem. Phys., Vol. 117, pp. 10139-10150, 2002.

[13] M. Rosenblit and J. Jortner, "Dynamics of the Formation of an electron bubble in liquid helium”, Phys. Rev. Lett., Vol. 75, pp. 4079-4083, 1995.

[14] A.P. Hickman, W. Steets and N.F. Lane, "Nature of excited atoms in liquid helium: a theoretical model", Phys. Rev. B, Vol. 12, pp. 3705$3717,1975$.

[15] L. Meyer, H.T. Davis, S.A. Rice, R.J. Donnelly, "Mobility of Ions in Liquid ${ }^{4} \mathrm{He}$ and ${ }^{3} \mathrm{He}$ as a Function of Pressure and Temperature", Phys. Rev., Vol. 126, pp. 1927-1934, 1962.

[16] J. Levine and T.M. Sanders, "Mobility of Electrons in Low-Temperature Helium Gas", Phys. Rev., Vol. 154, pp. 138-145, 1967.

[17] V.M. Atrazhev, "Delocalization of electrons in dense helium gas by external electric field", J. Phys. D, Appl. Phys., Vol. 17, pp. 889-902, 1984.

[18] Z. Li, N. Bonifaci, A. Denat, V.M. Atrazhev and V.V. Atrazhev, "Ionization and charge transport phenomena in liquid helium induced by corona discharge", J. Electrostatics, Vol. 66, pp. 263-274, 2008.

[19] A.B. Callear, R.E.M. Hedges, "Metastability of Rotationally Hot Dihelium at 77 K", Nature, Vol. 215, pp. 1267-1271, 1967.

[20] W.R. Hindmarsh, A.D. Petford and G. Smith, "Interpretation of collision broadening and shift in atomic spectra", Proc. Roy. Soc., Vol. A297, pp. 296-304, 1967.

[21] Z.-L. Li, Décharge Couronne dans l'hélium Liquide et Gas Dense sous Champ Electrique Intense: Pre-claquage, Transport de Charge, Spectroscopie d'emission, Ph.D. Thesis, J. Fourier University, Grenoble, France, 2008.

[22] H. Margenau, "Theory description of pressure shift and quenching of excited states in liquid helium", Phys. Rev., Vol. 45, pp. 755-765, 1935.

[23] J. P. Hansen and E. L. Pollock, "Liquid-helium configuration around a metastable excited helium atom”, Phys. Rev. A, Vol. 5, pp. 2214-2217, 1972.

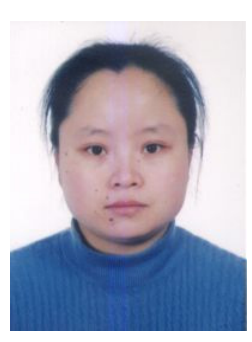

Zhiling Li was born in 1973 in the Guizhou province, China. She received the B.Sc. degree at the Chinese Institute of Metrology (Hangzhou, China) in 1997, the Master degree in physics at the Joseph Fourier University of Grenoble (France) in 2004 and the Ph.D. degree at the same institution in 2008. Presently, she works as a researcher at the University of Namur, Belgium. Her research interest are high electric field conduction and pre-breakdown phenomena in dielectric liquids.

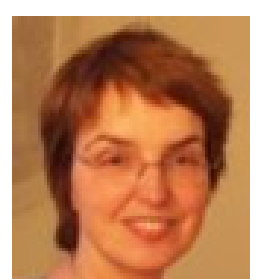

Nelly Bonifaci studied physics at the Joseph Fourier University in Grenoble where she achieved her Ph.D. degree in 1992 and subsequently joined the CNRS as a full researcher. She specialized there in investigations of cryogenic liquids and high-pressure gases (Nitrogen and Argon especially) using luminescence spectroscopy and corona-like discharges. She is also involved in environmental research, for instance, on the replacement of $\mathrm{SF}_{6}$ insulation in high-voltage devices.

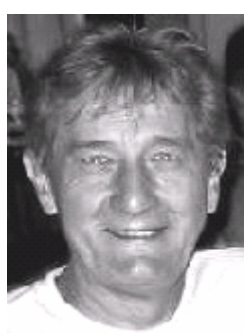

André Denat started his career in the chemical industry, and then joined the academic institution as an engineer. He obtained his Doctorate in physics (1982) and is now full-time researcher (Directeur de Recherches) at the Centre National de la Recherche Scientifique (CNRS). He was the head of the Laboratory for Electrostatics and Dielectric Materials (LEMD) in Grenoble from 1997 up to 2004. His fields of interest are the study of high field conduction phenomena and their influence on the initiation of pre-breakdown events: injection of charges at metalinsulating liquid interfaces, electron avalanches in insulating liquids and gases, spectroscopic analysis of discharges, phase transition induced by discharges, etc.

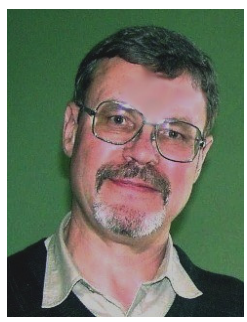

Vladimir M. Atrazhev was born in Moscow (Russia) in 1949. He graduated from the Department of Physics, Moscow State University in 1972. From 1972 to 2002 he was a Scientific Researcher in the Theoretical Department of the Institute for High Temperature (IHT) of Russian Academy of Sciences, where he obtained his Ph.D. degree in 1979. In 2002 Dr. Atrazhev became a Lead Researcher at the Institute for High Energy Densities within the Associated Institute for High Temperatures, Russian Academy of Sciences, Moscow (Russia). From 1992 till now he has been repeatedly invited to work as a visiting researcher at the Laboratoire d'Electrostatique et de Materiaux Dielectriques, CNRS and Fourier University, Grenoble (France). His research interests include transport properties of electrons in strong electric field in dense media, properties of non-equilibrium plasma and discharge, and electric breakdown of dense gases and liquid dielectrics.

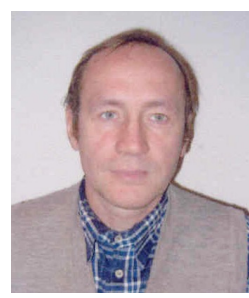

Vyacheslav A. Shakhatov was born in Moscow, Russian Federation in 1963. He graduated from the Department of Physics, Moscow Physico-Technical Institute, Russian Federation in 1986. From 1986 to 2003 he was a Scientific Researcher of the Moscow Radio-Technical Institute RAS, where he obtained his Ph.D. degree in 1997. From 2003 till now he is a Senior Staff Researcher of the A.V. Topchiev Institute of Petrochemical Synthesis RAS. His research interests include coherent anti-Stokes Raman spectroscopy, laser light scattering, emission spectroscopy and optical interferometry, and shadow visualization techniques of combustion and plasma systems, plasma physics and chemistry, new materials and processing, numerical simulation of the energy transfer in molecular aggregates and atomic species. 


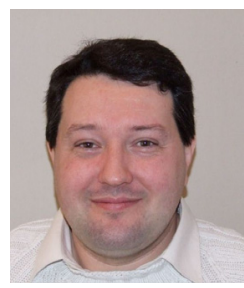

Frédéric Aitken obtained his master degree in physics at the Joseph Fourier University at Grenoble. There, he achieved his Ph.D. degree in 1998 and joined the CNRS as a full researcher shortly thereafter. $\mathrm{He}$ investigates fast energy injection in dielectric liquids for the characterization of phase changes induced by corona-like discharges. He also developed a theory on phase changes induced by electronavalanches.

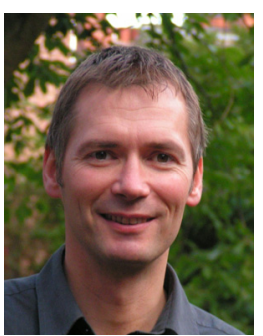

Klaus von Haeften graduated in physics at the University of Hamburg and achieved his Doctorate in 1999. He worked at the Max-Planck-Institute for Flow Research, the German Electron Synchrotron (DESY) and the Departments of Chemistry at the University of Bochum and the University of Leicester (UK). In 2006 he was appointed as a Nanoscience Lecturer at the Department of Physics \& Astronomy at the University of Leicester. His research interest include the physical and chemical properties of clusters and nano-structured materials and their application. Part of his work is devoted to the spectroscopy of pure and doped helium clusters and droplets. $\mathrm{He}$ is familiar with various spectroscopic techniques as well as laser and synchrotron light sources. 